\title{
A Mathematical Model of Dynamic Social Networks
}

\author{
John L. Pfaltz \\ Dept. of Computer Science, University of Virginia \\ jlp@virginia.edu
}

\begin{abstract}
A mathematical model for dynamic networks is developed that is based on closed, rather than open, sets. For a social network it seems appropriate to use a neighborhood concept to establish these sets. We then define a rigorous concept of continuous change, and show that it shares some of the properties associated with the continuity of the calculus.

We demonstrate that continuity is local in nature, in that if the network change is discontinuous it will be so at a single point and the discontinuity will be apparent in that point's immediate neighborhood.
\end{abstract}

Necessary and sufficient criteria for continuity are provided when the change involves only the addition, or deletion, of individual nodes or connections (edges).

To illustrate large scale continuous change we choose a practical process which reduces a complex network to its chordless cycles, in the course of which most triadically closed subportions are removed.

Finally, we explore several variants of the neighborhood concept, and prove that a rigorous notion of fuzzy closure can be defined.

Keywords: closed set, transformation, neighborhood, continuity, chordless cycle, graph reduction, triadic closure

\section{Introduction}

Networks, or undirected graphs (which we regard as total synonyms) are fundamental for modeling social phenomena [5]. Yet they also abound in both the sciences and humanities, c.f. [28] for its excellent survey and bibliography of over 400 applications. They may be huge; the connectivity of the world wide web is a network - they may be tiny; the atomic bonds in a molecule are an undirected graph. A representative example is the 379 node network of collaborating scientists constructed by M.E.J. Newman [29] illustrated in Figure 1, in which each edge denotes at least one co-authored paper. ${ }^{1}$ This will reappear in Section 4.1.

Such networks are dynamic. Yet, even though it is recognized that networks may arise from dynamic processes [6], there has been little formal study of network change itself $[3,12]$. This paper focuses on how a network can change, any network, but especially social networks. We introduce the concept of network transformation in Section 3. Typically, we are interested in those kinds of transformations which preserve elements of network structure. In particular, we are concerned with "continuous" transformations.

The calculus of continuous functions over manifolds is defined in terms of open sets. When the domains are finite discrete structures, closed sets

\footnotetext{
${ }^{1}$ Figure reprinted with permission from M.E.J.Newman, Phys. Rev. E 74, 036104 (2006). Copyright (2006) by the American Physical Society.
} 


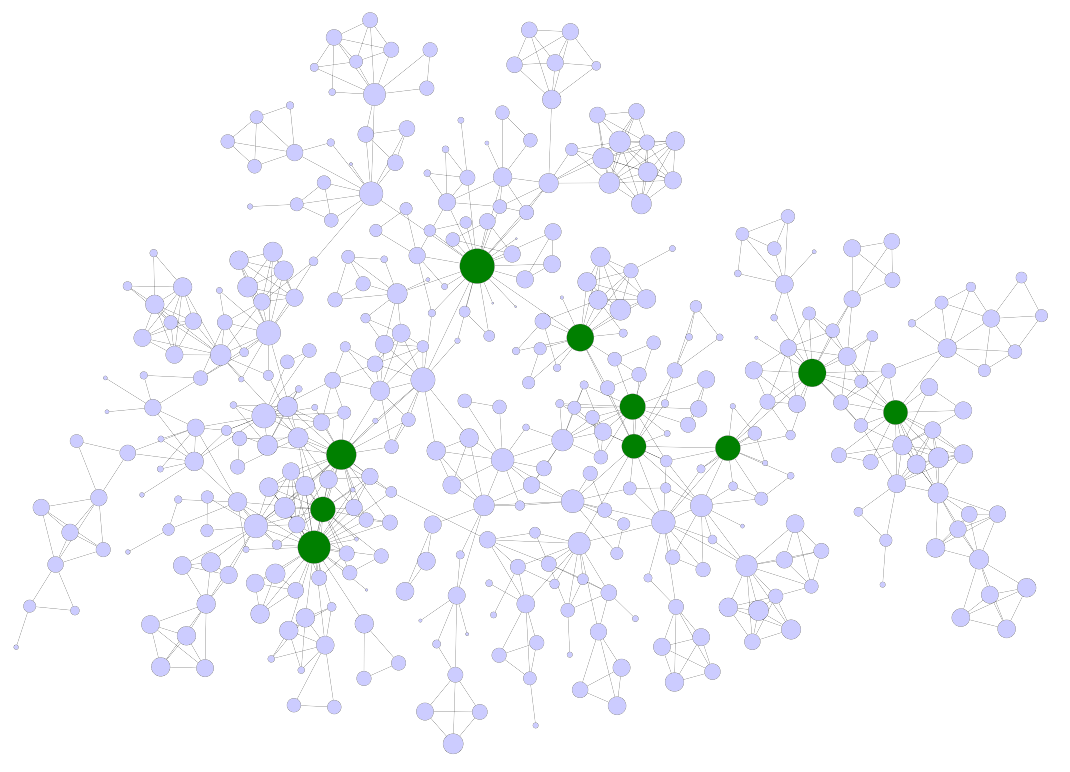

Figure 1: A 379 node network of collaborating scientists [29].

become a more powerful tool for their analysis. Closure is not a new concept in the social sciences. For example, it is associated with rational choice operators in economics $[20,27,26]$. Galois closure can be used to extract rules from data sets for subsequent used in A.I. reasoning systems $[34,35]$. When the system can be partially, or totally, ordered the closed sets are usually intervals, ideals or filters $[19,24]$. In this paper we employ the closed set structure of undirected graphs and networks.

Much of the current mathematical analysis of social networks is statistical $[22,40]$ or combinatoric [38]. Both can provide valuable, broadbrush properties of the entire system. In contrast, our approach focuses on the decomposition of the system into its constituent closed set structure. The closed sets are created by a neighborhood closure introduced in Section 2.1.

In Section 3, we define the concept of continuous transformations of discrete systems in general, and use it in Section 3.1 to explore the behavior of continuous network transformations. All of the mathematical results associated with network closure in these two sections are original.

Section 4.1 presents a representative graph reduction process that is applicable to large networks; it is shown to be continuous. Neighborhoods play a fundamental role throughout the paper; in section 4.2, 4.3 they are explored a bit more fully. Lastly, Section 4.4 introduces the notion of "fuzzy" closure.

\section{Closure}

An operator $\varphi$ is said to be a closure operator if for all $Y, Z \subseteq P$, it is:

(C1) extensive, $Y \subseteq Y . \varphi$, and,

(C2) monotone, $Y \subseteq Z$ implies $Y . \varphi \subseteq Z . \varphi$,

(C3) idempotent, $Y \cdot \varphi \cdot \varphi=Y \cdot \varphi$.

A subset $Y$ is closed if $Y=Y . \varphi$. In this work we prefer to use suffix notation, in which an operator follows its operand. Consequently, when operators are composed the order of application is read naturally from left to right. With this suf- 
fix notation read $Y . \varphi$ as " $Y$ closure". It is well known that the intersection of closed sets must be closed. This latter can be used as the definition of closure, with the operator $\varphi$ defined by $Y . \varphi=\bigcap_{Z_{i} \text { closed }}\left\{Y \subseteq Z_{i}\right\}$.

By a closure system $\mathcal{S}=(P, \varphi)$, we mean a set $P$ of "points" or "elements", together with a closure operator $\varphi$. By (C1) the set $P$ must be closed. In a social network these points are typically individuals, or possibly institutions. The empty set, $\varnothing$, may, or may not, be closed.

A point $y \in Y$ is said to be a ordinary point of $Y$ if $y \in(Y-\{y\}) . \varphi$. In contrast, a point $y \in$ $Y$ is said to be an extreme point of $Y$ if $y \notin$ $(Y-\{y\}) \cdot \varphi$. (Extreme points have a central role in antimatroid closure theory $[2,8]$.) $\mathrm{A}$ set is said to be whole if all of its points are ordinary points.

\subsection{Neighborhood Closure}

Let $\mathcal{S}=(P, \mathcal{A})$ be a set $P$ of points, or elements, together with a symmetric adjacency relation $\mathcal{A}$. By the neighborhood, or neighbors, of a set $Y$ we mean the set $Y . \eta=\{x \notin Y \mid \exists y \in Y,(x, y) \in$ $\mathcal{A}\}$. Note that this is a purely formal definition of "neighborhood", independent of the social aspects often implied by the term [17]. By the region dominated by $Y$ we mean $Y . \rho=Y \cup Y . \eta .^{2}$ Suppose $P$ is a set of individuals and the relation $\mathcal{A}$ denotes a symmetric connection, such as mutual communication, between them. The neighborhood $y . \eta$ about a person $y$ is the set of individuals with which $y$ directly communicates. The neighborhood, $Y . \eta$, of a set $Y$ of individuals is the set of individuals not in $Y$ who directly communicate with at least one individual in $Y$. The region, $Y . \rho$, also includes $Y$ itself. Members of $Y$ may, or may not, communicate with each other. Unfortunately, knowledge of the region $Y . \rho$ dominated by a set $Y$ reveals very little about $Y$ itself. For example, in Figure 2 we

\footnotetext{
${ }^{2}$ In graph theory, $Y . \eta$ is often called the "open neighborhood of $Y$ " and denoted $N(Y)$, while $Y . \rho$, denoted $N[Y]$ has been called the "closed neighborhood of $Y$ " $[1,15]$. This is a rather different meaning of "closed".
}

have $\{b d\} \cdot \rho=a b c d g \subseteq$ abcdefgh $=\{c g\} . \rho$, yet $\{b d\} \cap\{c g\}=\varnothing$. There is an extensive literature regarding dominating sets, $c . f$. [16].

We can visualize the neighborhood structure of a discrete set of points, or individuals, as an undirected graph such as Figure 2. The neighbors of any point are those adjacent in the graph. In

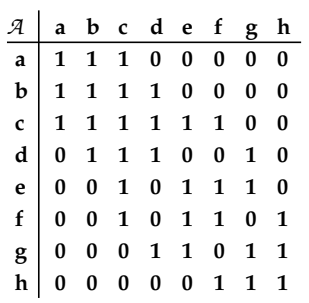

(a)

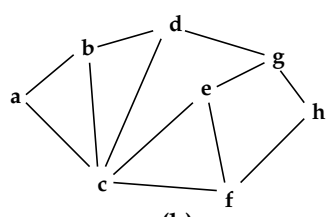

(b)

Figure 2: A symmetric adjacency matrix $\mathcal{A}$ and corresponding undirected graph.

the graph of Figure 2 we have $\{a\} \cdot \eta=\{b, c\}$ or more simply $a . \eta=b c$. And $g . \rho=d e g h$.

Given the neighborhood concepts $\eta$ and $\rho$, we define the neighborhood closure, $\varphi_{\eta}$ to be

$$
Y . \varphi_{\eta}=\{x \mid x . \rho \subseteq Y . \rho\}
$$

In a social system, the closure of a group $Y$ of individuals are those additional individuals, $x$, all of whose connections match those of the group $Y$. A minimal set $X \subseteq Y$ of individuals for which $X . \varphi_{\eta}=Y . \varphi_{\eta}$ is sometimes called the nucleus, core, or generator of $Y . \varphi_{\eta}$. Readily, for all $Y$,

$$
Y \subseteq Y . \varphi_{\eta} \subseteq Y . \rho
$$

that is, $Y$ closure is always contained in the region dominated by $Y$.

Proposition 2.1 $\varphi_{\eta}$ is a closure operator.

Proof: (C1) Readily, $Y \subseteq Y . \varphi_{\eta}$ by definition. (C2) Let $X \subseteq Y$ and let $z \in X . \varphi_{\eta}$. By (1) $z . \rho \subseteq$ 
$X . \rho \subseteq Y . \rho$ hence $z \in Y . \varphi_{\eta}$.

(C3) Let $z \in Y \cdot \varphi_{\eta} \cdot \varphi_{\eta}$. Then $z . \rho \subseteq Y \cdot \varphi_{\eta} \cdot \rho=$ $\bigcup_{x \in Y . \varphi_{\eta}} x . \rho \subseteq Y . \rho$, hence $z \in Y . \varphi_{\eta}$.

Proposition $2.2 X . \varphi_{\eta} \subseteq Y . \varphi_{\eta}$ if and only if $X . \rho \subseteq Y . \rho$.

Proof: Let $X . \varphi_{\eta} \subseteq Y . \varphi_{\eta} . \forall x \in X . \varphi_{\eta}, x . \rho \subseteq X . \rho$, so $x \in Y . \varphi_{\eta}$ implies $x . \rho \subseteq Y$. $\rho$ or $X . \rho \subseteq Y$. $\rho$.

Now suppose $X . \rho \subseteq Y . \rho$. Let $z \in X . \varphi_{\eta}$ implying $z . \rho \subseteq X . \rho \subseteq Y . \rho$ Hence $z \in Y . \varphi_{\eta}$.

Corollary $2.3 X . \varphi_{\eta}=Y . \varphi_{\eta}$ if and only if $X . \rho=Y . \rho$.

Proposition 2.4 Let $\varphi_{\eta}$ be the closure operator. If $y . \eta \neq \varnothing$ then there exists $X \subseteq y . \eta$ such that $y \in X . \varphi_{\eta}$.

Proof: Readily, $y . \rho \subseteq y . \eta . \rho$, so $y \in y . \eta . \varphi_{\eta}$. Choose a minimal $X \subseteq y . \eta$ such that $X . \rho \subseteq y . \rho$.

So, unless $y$ is an isolated point, every point $y$ is in the closure of some subset of its neighborhood.

One might expect that every point in a discrete network must be closed, e.g. $\{x\} \cdot \varphi_{\eta}=\{x\}$. But, this need not be true, as shown in Figure 2. The region $c . \rho=a b c d e f$ while $a . \rho=a b c \subseteq c . \rho$ and $b . \rho=a b c d \subseteq c . \rho$, so $c . \varphi_{\eta}=a b c$. The points $a$ and $b$ are ordinary points of $Y=\{a b c\}$, but $Y$ is not whole because $c \notin(a b c-c) \cdot \varphi=\{a b\}$.

Equation (2) suggests an effective computer algorithm to calculate the closure $Y \cdot \varphi_{\eta}$ of any set $Y$. Initially, let $Y . \varphi_{\eta}=Y$; then since $Y . \rho=$ $Y \cup Y . \eta$ examine only the points $z$ in the neighborhood, $Y . \eta$, of $Y$. If $z . \rho \subseteq Y$. $\rho$, add $z$ to $Y . \varphi_{\eta}$.

Proposition 2.5 If $\varphi_{\eta}$ is the closure operator and $y$ is an ordinary point of $Y$, then $y . \rho \subseteq$ $(Y-\{y\}) . \rho \subseteq Y . \rho$.

Proof: The first containment follows from the definition of $y \in(Y-\{y\}) \cdot \varphi_{\eta}$. The second containment is always true.
Proposition 2.6 Let $\varphi_{\eta}$ be the closure operator. If $Y$ is whole then $Y$ is closed.

Proof: Suppose $Y$ is not closed, implying $\exists y \in Y$. $\rho$, $y . \rho \nsubseteq Y . \rho$. Then, by Prop. 2.5, y cannot be an ordinary point, so $Y$ cannot be whole.

Of course, closed sets need not be whole.

Proposition 2.7 Let $\varphi_{\eta}$ be the closure operator. If $X$ and $Y$ are finite whole sets and $X \cap Y \neq \varnothing$, then $X=Y$.

Proof: Let $z \in X \cap Y$, so $z$ is an ordinary point of both $X$ and $Y$. By Prop. 2.5, z. $\subseteq X . \rho \cap Y . \rho$. Consequently the iterated neighborhood z. $\rho \ldots \subseteq$ $X \rho \ldots \rho \cap Y \rho \ldots \rho$, and since both are finite this iteration must terminate with $X \subseteq X \cap Y, Y \subseteq X \cap Y$, so $X=Y=X \cap Y$.

It is apparent that with respect to neighborhood closure, whole sets are effectively the nontrivial connected components of the network.

\section{Transformations}

Social processes create corresponding social networks. Ongoing social precesses also transform these networks in ways that can reveal the nature of the processes themselves.

We use graph-theoretic concepts to model social networks; but graph theory provides scant models for describing their change. Almost any book on graph theory mentions graph homomorphism, that is a mapping $h:(P, E) \rightarrow\left(P^{\prime}, E^{\prime}\right)$, or a function $h: P \rightarrow P^{\prime}$ in which $(x, y) \in E$ implies that $(h(x), h(y)) \in E^{\prime}[1,15] .{ }^{3}$ But, a serious limitation of graph homomorphisms is that, since $h: P \rightarrow P^{\prime}$ is a function, the homomorphic "image" must always be "smaller". We call this the "curse of cardinality" when mathematically modelling finite, discrete systems. In the real world, dynamic networks can both expand and contract.

\footnotetext{
${ }^{3}$ Because homomorphisms are point functions, not set valued operators, we employ prefix notation.
} 
For this reason we introduce the notion of a graph, or network, transformation which is a function mapping the power set, $2^{P}$, of $P$ into the the power set, $2^{P^{\prime}}$, of $P^{\prime}$. That is, every subset of $P$ has a unique image subset in $P^{\prime}$. The operators $\eta, \rho$, and $\varphi_{\eta}$ are therefore transformations of a network $(P, E)$ into itself, since every subset has a unique image. To emphasize this difference, a transformation $f$ is denoted by our suffix notation, e.g. Y.f, rather than the customary prefix notation of functions and homomorphisms. In neighborhood notation, a graph homomorphism $h$ would be characterized by y. $. h \subseteq y . h . \rho^{\prime}$.

We denote transformations of network systems by $(P, E) \stackrel{f}{\longrightarrow}\left(P^{\prime}, E^{\prime}\right)$, or possibly by $(P, \varphi)$ $\stackrel{f}{\longrightarrow}\left(P^{\prime}, \varphi^{\prime}\right)$, since we are often interested in the closure structure induced by the neighborhood system. Note that a transformation $f$ may only change the neighborhood system of $P$ and hence $\varphi^{\prime}$.

In this paper we require that all transformations $f$ be monotone, that is

$$
X \subseteq Y \text { implies } X . f \subseteq Y . f
$$

as seems to be normally the case in real applications. Note that "monotone" in this sense only preserves set containment relationships; it does not mean that the transformation is "increasing" or "decreasing". A transformation $f$ is said to be surjective if for every closed set $Y^{\prime}$ in $P^{\prime}$ there exists a subset $Y \subseteq P$ such that $Y . f=Y^{\prime} . Y$ need not be closed.

By convention $[31,32,42]$, a transformation $f$ is said to be continuous if for all $Y \subseteq P$

$$
Y . \varphi . f \subseteq Y . f . \varphi^{\prime}
$$

Readily, (4) holds for all closed sets $Y$ because $Y . \varphi . f=Y . f \subseteq Y . f . \varphi^{\prime}$.

If one visualizes $\varphi$ to be an operative force that causes social cohesion, then "continuity" assures that cohesion observed in the pre-image network will be contained in the cohesion modeled in the resulting image network. These first propositions are true for all closure operators, $\varphi$. In Section 3.1 we specifically address $\varphi_{\eta}$.
Proposition 3.1 Let $(P, \varphi) \stackrel{f}{\longrightarrow}\left(P^{\prime}, \varphi^{\prime}\right)$, $\left(P^{\prime}, \varphi^{\prime}\right) \stackrel{g}{\longrightarrow}\left(P^{\prime \prime}, \varphi^{\prime \prime}\right)$ be transformations and let $g$ be monotone. If both $f$ and $g$ are continuous, then so is $P \stackrel{f . g}{\longrightarrow} P^{\prime \prime}$.

Proof: We have $X . \varphi . f \subseteq X . f . \varphi^{\prime}$ for any $X \in P$ and $Y . \varphi^{\prime} . g \subseteq Y . g . \varphi^{\prime \prime}$ for any $Y \in P^{\prime}$. Consequently,

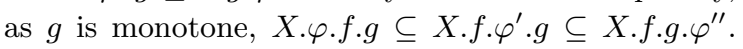
Thus $f \cdot g$ is continuous.

Continuous transformations of discrete spaces exhibit many of the properties of continuous real functions with which we are more familiar. For example, let $f$ be a function $f: R \rightarrow R^{\prime}$; if (a) $f$ is onto, then for all $y^{\prime} \in R^{\prime}$ there exists $y \in R$ such that $f(y)=y^{\prime}$; if (b) $f$ is continuous and $X^{\prime}$ is open/closed in $R^{\prime}$, then $f^{-1}\left(X^{\prime}\right)$ is open/closed in $R$.

Proposition 3.2 Let $(P, \varphi) \stackrel{f}{\longrightarrow}\left(P^{\prime}, \varphi^{\prime}\right)$ be monotone, continuous and let $Y^{\prime}=Y . f$ be closed. Then Y. $\varphi . f=Y^{\prime}$.

Proof: Let $Y . f$ be closed in $P^{\prime}$. Because $f$ is continuous $Y . \varphi . f \subseteq Y . f . \varphi^{\prime}=Y . f$, since $Y . f$ is closed. By monotonicity, $Y$.f $\subseteq Y . \varphi . f$, so $Y . \varphi . f=Y$.f.

Or, in effect, if the pre-image of a closed set exists it must also be, in a sense, closed. However, if a continuous $f$ is also surjective then every closed set $Y^{\prime}$ in $P^{\prime}$ has at least one closed set, $Y$, such that $Y . f=Y^{\prime}$, and $f^{-1}$ is well defined over the closed sets of $P^{\prime}$.

One can also consider $\varphi$-preserving transformations which map closed sets in $P$ onto closed sets in $P^{\prime}{ }^{4}$ It is apparent that the composition of $\varphi$-preserving transformations is $\varphi$-preserving.

Proposition 3.3 A monotone transformation $(P, \varphi) \stackrel{f}{\longrightarrow}\left(P^{\prime}, \varphi^{\prime}\right)$ is $\varphi$-preserving if and only if $\forall X \subseteq P, \quad X . f . \varphi^{\prime} \subseteq X . \varphi . f$.

Proof: Let $f$ be $\varphi$-preserving. By monotonicity, $X \subseteq X . \varphi$ implies $X . f \subseteq X . \varphi$.f. But, because $X . \varphi$ is

\footnotetext{
${ }^{4}$ The topological term "closed" is traditional for structure preserving maps, whether expressed in terms of open sets or closed sets. But, it is most unfortunate in this context, where the multiple meanings can lead to confusion.
} 
closed and $f$ is $\varphi$-preserving, $X . f . \varphi^{\prime} \subseteq X . \varphi . f$ Conversely, let all subsets $X \subseteq P$ fulfill $X . f . \varphi^{\prime} \subseteq$ $X . \varphi . f$ and let $X$ be a closed subset of $(P, \varphi)$. Then $X . f . \varphi^{\prime} \subseteq X . f$. But, readily $X . f \subseteq X . f . \varphi^{\prime}$, so equality holds.

Consequently,

Proposition 3.4 A monotone transformation $(P, \varphi) \stackrel{f}{\longrightarrow}\left(P^{\prime}, \varphi^{\prime}\right)$ is $\varphi$-preserving and continuous if and only if, for all $X \subseteq P, X . \varphi \cdot f=$ $X . f \cdot \varphi^{\prime}$.

A common way of defining a graph transformation $(P, E) \stackrel{f}{\longrightarrow}\left(P^{\prime}, E^{\prime}\right)$ is to first define $\{y\} . f$ for all singleton sets in $P$ and then extend this to all $Y \subseteq P$ by $Y . f=\bigcup_{y \in Y}\{y\} . f$. We call $f$ an extended transformation if $P . f=P^{\prime}$. Any extended transformation is by construction, monotonic.

Proposition 3.5 If $(P, E) \stackrel{f}{\longrightarrow}\left(P^{\prime}, E^{\prime}\right)$ is an extended transformation, then for all $y^{\prime} \in Y^{\prime}=$ $Y$.f there exists $y \in Y$ such that $y^{\prime} \in\{y\} . f$.

Proof: Let $y^{\prime} \in Y^{\prime}$. By the extended construction $Y^{\prime}=\bigcup_{y \in Y}\{y\} . f$, hence $y^{\prime} \in\{y\} . f$ for some $y \in Y$.

Note that this is quite different from asserting a true inverse existence, that for all $y^{\prime} \in Y^{\prime}$, there exists some $y \in Y$ such that $y . f=y^{\prime}$. To get some sense of the import of this "weak inverse existence" proposition, consider the simple transformation $f$ of Figure 3 . If we define $f$ on

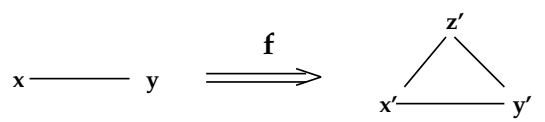

Figure 3: A simple transformation $f$ with multiple definitions.

$P$ by $x . f=x^{\prime}$ and $y . f=y^{\prime}$, then by extension $\{x y\} . f=x^{\prime} y^{\prime}$ and $z^{\prime}$ has no pre-image; so P.f $\neq$ $P^{\prime}$. However, if we let $x . f=\left\{x^{\prime} z^{\prime}\right\}, y . f=\left\{y^{\prime} z^{\prime}\right\}$ then $\{x y\} . f=x^{\prime} y^{\prime} z^{\prime}$. Now P.f $=P^{\prime}$, so $f$ is an extended transformation, and Proposition 3.5 is clearly satisfied. Whether or not this $f$ is surjective depends on the closure structures of $P$ and $P^{\prime}$.

Unless otherwise explicitly stated, all remaining examples of this paper will be extended transformations.

\subsection{Network Transformations}

The preceding mathematical assertions are true for all closure systems. Now we focus specifically on network transformations. It is the neighborhood, $y . \eta$, which is central.

Proposition 3.6 Let $x \in y . \eta$, then $x \in y \cdot \varphi_{\eta}$ if and only if $x . \rho \subseteq y . \rho$ if and only if $x . \eta-\{y\} \subseteq$ $y . \eta$.

Proof: The first equivalence is simply a restatement of the definition of neighborhood closure. The second equivalence follows because if $x . \rho \subseteq y . \rho$ then $\forall z \neq y$, $z \in x . \eta$ implies $z \in y . \eta$. The converse is similar.

Proposition 3.7 Let $(P, E) \stackrel{f}{\longrightarrow}\left(P^{\prime}, E^{\prime}\right)$ be extended. If $f$ is not continuous, there exists $Y \subseteq$ $P$, and $y \in Y . \eta$ such that either

(1) $y^{\prime} \notin Y . f . \eta^{\prime}$

or

(2) $y . \eta \subseteq Y . \eta$ and $y^{\prime} . \eta^{\prime} \nsubseteq Y . f . \eta^{\prime}$

Proof: Since $f$ is not continuous, there exists $Y$ such that $Y . \varphi_{\eta} . f \nsubseteq Y . f . \varphi_{\eta^{\prime}}^{\prime}$. Thus, $\exists y^{\prime} \in Y . \varphi_{\eta} . f, y^{\prime} \notin$ $Y . f . \varphi_{\eta^{\prime}}^{\prime}$. By, Prop. 3.5, $\exists y \in Y . \varphi_{\eta}$ such that $y^{\prime} \in y . f$. $y \notin Y$ else $y^{\prime} \in Y$.f. Consequently, $y \in Y . \eta$ and $y . \eta \subseteq Y . \eta$. Now, since $y^{\prime} \notin Y . f . \varphi_{\eta^{\prime}}^{\prime}$ we know that either $y^{\prime} \notin Y . f . \eta^{\prime}$ or $y^{\prime} . \eta^{\prime} \nsubseteq Y . f . \eta^{\prime} . Y$ is technically unspecified, but by Prop. $3.6 y \in y . \eta \cdot \varphi_{\eta}$; hence we can assume $\{y\} \subseteq Y \subseteq y . \eta$.

This proposition establishes that if $f$ is discontinuous anywhere, then it will be discontinuous at, or near, a point $y$. One need not consider all subsets of $2^{P}$. Just as is the case with classical function theory, discontinuity, and thus continuity, is a local phenomena. Secondly, it provides conditions (1) and (2) which are are necessary, but not sufficient to demonstrate discontinuity. If for a point $y \in P$ neither condition (1) nor (2) 
holds, we say $f$ is continuous at $y$. If either condition holds, other criteria must be used, $c . f$. propositions $3.8,3.9$ or 3.12 .

We have said that a transformation $P \stackrel{f}{\longrightarrow} P^{\prime}$ is monotone if $\forall X, Y, X \subseteq Y$ implies $X$.f $\subseteq Y$. $f$. Let $(P, E) \stackrel{f}{\longrightarrow}\left(P^{\prime}, E^{\prime}\right)$ be a transformation between two neighborhood systems. The transformation $f$ is said to be neighborhood monotone if $X . \rho \subseteq Y . \rho$ implies $X . f . \rho^{\prime} \subseteq Y . f . \rho^{\prime}$. A transformation that is monotone need not be neighborhood monotone, and conversely.

Proposition 3.8 Let $(P, E) \stackrel{f}{\longrightarrow}\left(P^{\prime}, E^{\prime}\right)$ be monotone, then $f$ is continuous if and only if $f$ is neighborhood monotone.

Proof: Let $f$ be continuous and let $X . \rho \subseteq Y$. $\rho$. By Prop. 2.2, $X \subseteq X . \varphi \rho \subseteq Y . \varphi \rho$. Thus, $X . f \subseteq$ $Y . \varphi \rho . f \subseteq Y . f . \varphi_{\rho}^{\prime}$ by continuity. So $X . f . \rho^{\prime} \subseteq Y . f . \rho^{\prime}$. Conversely, let $f$ be neighborhood monotone. By definition $Y . \varphi_{\eta}=Y \cup\{x \notin Y \mid x . \rho \subseteq Y . \rho\}$. Since for all $y \in Y, y^{\prime} \in Y . f \subseteq Y . f . \varphi_{\eta}{ }^{\prime}$, we need only consider $x \notin Y$, but $x . \rho \subseteq Y$. $\rho$. Since $f$ is neighborhood monotone, $x . \rho \subseteq Y . \rho$ implies $x^{\prime} . \rho^{\prime}=x . f . \rho^{\prime} \subseteq Y . f . \rho^{\prime}$ so $x^{\prime} \in Y . f . \varphi_{\eta}{ }^{\prime}$.

\section{$3.2 \quad$ Network Growth}

Unfortunately, both propositions 3.7 and 3.8 can be awkward to use in practice. We look for a more local criteria.

A network can grow by adding points and/or edges. Any transformation which just adds an isolated point $z^{\prime}$ will be continuous, since if $X$ is closed in $(P, \varphi), X^{\prime}$ and $X^{\prime} \cup\left\{z^{\prime}\right\}$ will be closed in $\left(P^{\prime}, \varphi^{\prime}\right)$. But, if continuity is important, care must be taken when adding edges or connections.

Proposition 3.9 An extended network transformation $f$, which adds an edge $\left(x^{\prime}, z^{\prime}\right)$ to $\mathcal{A}^{\prime}$ at $x$, will be continuous at $x$ if and only if $x \in Y . \varphi_{\eta}$, $Y \subseteq x . \eta^{6}$ implies $z \in Y . \eta$.

Proof: First we note that unless $x$ is isolated, by Prop. 2.4, there does exist a set $Y \subseteq x . \eta$ such that $x \in Y \cdot \varphi_{\eta}$.
If the condition does not hold, i.e. $z \notin Y . \eta$ then $z^{\prime} \in$ $x^{\prime} . \eta^{\prime}$ ensures that $x \notin Y . f . \varphi_{\eta}{ }^{\prime}$ so $Y . \varphi_{\eta} . f \nsubseteq Y . f . \varphi_{\eta}{ }^{\prime}$. $f$ is not continuous.

Conversely, if $z \in Y . \eta$ then $x^{\prime} \in Y . f . \varphi_{\eta}{ }^{\prime}$ and Y. $\varphi . f \subseteq$ $Y . f . \varphi_{\eta}^{\prime}$ and $f$ will be continuous.

Readily, the same conditions must apply to $z$ as well.

If the connections/edges are between individuals, as in social networks, then Proposition 3.9 asserts that creating a connection $(x, z)$ between two persons, $x$ and $z$ where $x$ is closely bound to a set of individuals $Y$ is smoother, easier, or continuous if a connection already exists between $Y$ and $z$. This seems to be the case in numerous studies cited by [5]. The transformation $f_{1}$ in Figure 4 which adds the edge $\left(c^{\prime}, f^{\prime}\right)$ to $G_{1}$ satisfies Prop. 3.9 because $c \in\{b c\} . \varphi_{\eta}$, but $f \in e . \varphi_{\eta}$ as well.

Expansion of $G_{2}$ at $a^{\prime}$ by creating the edge $\left(a^{\prime \prime}, j^{\prime \prime}\right)$ is different. Because $a^{\prime} \in b^{\prime} \cdot \varphi_{\eta^{\prime}}^{\prime}$ (and $\left.c^{\prime} \cdot \varphi_{\eta^{\prime}}^{\prime}\right)$, but $\left(b^{\prime}, j^{\prime}\right) \notin \mathcal{A}^{\prime}$, by Prop. $3.9 f_{2}$ is discontinuous at $b$ (and also $c$ ). We would also observe that $f_{2}$ is not neighborhood monotone at $b^{\prime}$ because $a^{\prime} \cdot \eta^{\prime}=a^{\prime} b^{\prime} c^{\prime} \subseteq b^{\prime} \cdot \eta^{\prime}=a^{\prime} b^{\prime} c^{\prime} d^{\prime}$ but $a^{\prime \prime} \cdot \eta^{\prime \prime}=a^{\prime \prime} b^{\prime \prime} c^{\prime \prime} j^{\prime \prime} \nsubseteq b^{\prime \prime} \cdot \eta^{\prime \prime}=a^{\prime \prime} b^{\prime \prime} c^{\prime \prime} d^{\prime \prime}$, so $f_{2}$ is not continuous by Prop. 3.8 as well. Finally, we verify that $b^{\prime} \cdot \varphi_{\eta^{\prime}}^{\prime} \cdot f_{2}=a^{\prime \prime} b^{\prime \prime} \nsubseteq b^{\prime \prime}=b^{\prime \prime} \cdot \varphi_{\eta^{\prime \prime}}^{\prime \prime}$. As this example illustrates, the discontinuity need not occur at either $x$ or $z$, but often at some point $y$ in $x . \eta$ or $z . \eta$

Lastly, we make a more complex addition of two edges to the new point $y^{\prime \prime \prime}$ in $G_{4}$, viz. $\left(d^{\prime \prime \prime}, y^{\prime \prime \prime}\right)$ and $\left(g^{\prime \prime \prime}, y^{\prime \prime \prime}\right)$. This transformation $f_{3}$ satisfies Proposition 3.9. For example $d^{\prime \prime} \in$ $\left\{b^{\prime \prime} g^{\prime \prime}\right\} \cdot \varphi_{\eta}^{\prime \prime}$ (as well as $\left.\left\{c^{\prime \prime} g^{\prime \prime}\right\} \cdot \varphi_{\eta}^{\prime \prime}\right)$, so the inclusion of $\left(y^{\prime \prime \prime}, g^{\prime \prime \prime}\right)$ in $G_{4}$ by $f_{3}$ satisfies the necessary condition. While $f_{3}$ is continuous, a transformation that added only $\left(d^{\prime \prime \prime}, y^{\prime \prime \prime}\right)$ or $\left(g^{\prime \prime \prime}, y^{\prime \prime \prime}\right)$ would not be. We observe that $f_{3}$ is not a closed transformation because $\left\{d^{\prime \prime} g^{\prime \prime}\right\}$ is closed in $G_{3}$, but $\left\{d^{\prime \prime \prime} g^{\prime \prime \prime}\right\}$ is not closed in $G_{4}$ because $\left\{d^{\prime \prime \prime} g^{\prime \prime \prime}\right\} \cdot \varphi_{\eta}=\left\{d^{\prime \prime \prime} g^{\prime \prime \prime} y^{\prime \prime \prime}\right\}$. 


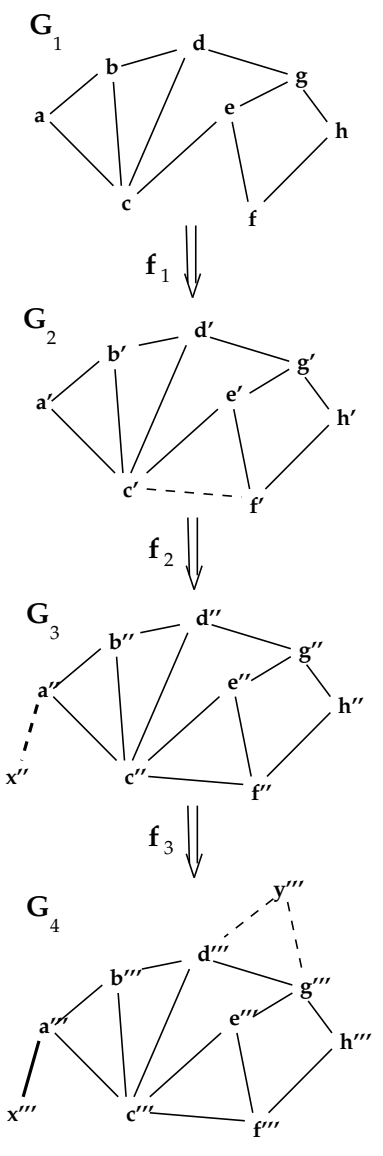

Figure 4: Three additive network transformations, $f_{1}, f_{2}$ and $f_{3}$.

\subsection{Triadic Closure}

Creating a relationship, or edge $(x, z)$, will be continuous if $x$ and $z$ are already connected, that is, there exist $y \in x . \eta$ and $y \in z . \eta$. The creation of $(x, z) \in \mathcal{A}$ is commonly known as triadic closure The transformation $f_{1}$ in Figure 4 is a classic example of triadic closure. The study of triads was initiated by Granovetter in [13], although he did not use the term "closure". It is not truly a closure operator (it is not idempotent); however, it appears to be a frequently occurring process in dynamic social systems $[14,21,25,30]$.
Corollary 3.10 Let $x, z \in y . \eta$, triadic closure adding $(x, z)$ will be continuous at $y$.

\subsection{Network Contraction}

Real networks lose members and connections; but this can be hard to model mathematically with homomorphic functions. The problem is that every point in the existing network must map to some point in the image space - and to be a homomorphism it must bring its edges/connections with it. Of course, if the two network elements are truly combined in reality then homomorphism is the right model. But, when the member or connection simply disappears, it isn't.

When we use the transformation model presented in this paper we can map a point, or subset, onto the empty set, $\varnothing$. We call it point removal. Removal of any point, or node $z$, must also delete all edges incident to $z$, that is all edges of the form $(y, z) \in E$. This is equivalent to deleting a row and column from the adjacency relation, $\mathcal{A}^{\prime}$. We let $\delta_{z}$ denote the removal of $z$ from $P^{\prime}$ and $(y, z)$ from $E^{\prime}$ for all $y \in z . \eta$.

Proposition $3.11 \delta_{z}$ is continuous at all $y \in$ $z . \eta$.

Proof: $\quad$ Let $X . \rho \subseteq Y . \rho . \quad$ Readily, $X . \rho-\{z\} \subseteq$ $Y . \rho-\{z\}$, so $X . \rho . \delta_{z} \subseteq Y . \rho . \delta_{z}$ and by Prop. 3.8, $\delta_{z}$ is continuous.

Instead of deleting a point and all its incident edges we can remove one, or more, connections thus changing the neighborhood structure represented by $\mathcal{A}^{\prime}$.

Proposition 3.12 An extended transformation $f$, which deletes a symmetric edge $(x, z)$ from $\mathcal{A}$ will be discontinuous if and only if either

(a) $z \in x . \varphi_{\eta}$ (or $x \in z . \varphi_{\eta}$ ), but $x . \varphi_{\eta} \neq z . \varphi_{\eta}$, or

(b) $(x, z)$ is an edge in a chordless cycle < $v, \ldots, w, x, z, \ldots, v>$ where either $|x . \eta|=2$ or $|z . \eta|=2$.

Proof: Suppose (a) holds and $z \in x . \varphi_{\eta}$. Then $z^{\prime}=z . f \in\{x\} . \varphi_{\eta} . f$, but $z^{\prime} \notin x . f . \varphi_{\eta}{ }^{\prime}$, so $f$ is 
discontinuous.

Suppose (b) holds and $|x \cdot \eta|=2$, so $x . \eta=\{w, z\}$. Now $x \in\{w, z\} . \varphi_{\eta}$ so $x^{\prime}=\{x\} . f \in\{w z\} . \varphi_{\eta} . f$, but $x^{\prime}=x . f \notin\{w z\} . f . \varphi_{\eta}{ }^{\prime}$ because $x \notin\left\{w^{\prime} z^{\prime}\right\} . \eta^{\prime}$ Again $f$ is discontinuous. The reasoning is similar when $x . \eta=\{w z\}$.

Conversely, suppose $f$ is discontinuous. Let $Y$ be a minimal set such that $Y . \varphi_{\eta} . f \nsubseteq Y . f . \varphi_{\eta}{ }^{\prime}$. Readily, either $z \in Y . \varphi_{\eta}$ but $z^{\prime}=z . f \notin Y . f . \varphi_{\eta}{ }^{\prime}$, (or $x \in Y . \varphi_{\eta}, x^{\prime}=x . f \notin Y . f . \varphi_{\eta}{ }^{\prime}$ ). We may assume the former. If $z \in Y$ then $z^{\prime} \in Y . f . \varphi_{\eta}{ }^{\prime}$ trivially, so $z \in Y . \eta$. Moreover, $z \in Y . \varphi_{\eta}$ implies $z . \eta \subseteq Y . \rho$. Since $(x, z) \in \mathcal{A}, z \in x . \eta$, thus $x \in Y$. If $Y=\{x\}$, then (a) holds and we are done.

Assuming $z \notin x . \varphi_{\eta}$ there must exist $v \in z . \eta, v \notin x . \eta$. Since $z \in Y \cdot \varphi_{\eta}, \exists w \in Y, v \in w . \eta$. We claim this cycle $\langle v, w, x, z, v\rangle$ is chordless. $v \notin x . \eta$ because $z \notin x . \varphi_{\eta} . z \notin w . \eta$ because $Y$ is minimal.

In social terms, Proposition 3.12 would assert that breaking a connection between $x$ and $z$ represents a discontinuity if $z$ is tightly bound to $x$, that is has the same shared connections to others nearby. This certainly seems to be true in the real world.

The second half of condition (a), $x \cdot \varphi_{\eta} \neq z \cdot \varphi_{\eta}$, is needed only for situations such as that of Figure 5 in which $x \cdot \varphi_{\eta}=z \cdot \varphi_{\eta}$ regardless of what

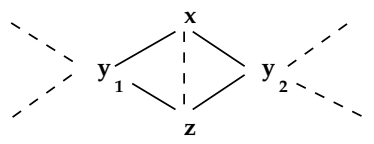

Figure 5: Two points with $x \cdot \varphi_{\eta}=z \cdot \varphi_{\eta}$.

other nodes are connected to $y_{1}$ and $y_{2}$. Addition, or deletion, of the dashed edge $(x, z)$ makes no change in the closed set structure whatever.

The transformations $f_{3}$ and $f_{4}$ of Figure 6 illustrate network contractions. In Figure 6 , the dashed edges of $G_{i}$ indicate the deletions in $G_{i+1}$.

By Prop. 3.12, removing the edge $(a, b)$ from $G_{1}$ is discontinuous because $b \in a \cdot \varphi_{\eta}$. Indeed, we find that $a \cdot \varphi_{\eta} \cdot f_{1}=a^{\prime} b^{\prime} \nsubseteq a \cdot f_{1} \cdot \varphi_{\eta^{\prime}}^{\prime}=a^{\prime}$. However, $f_{1}$ is continuous at $c \in a . \eta$. The transfor-
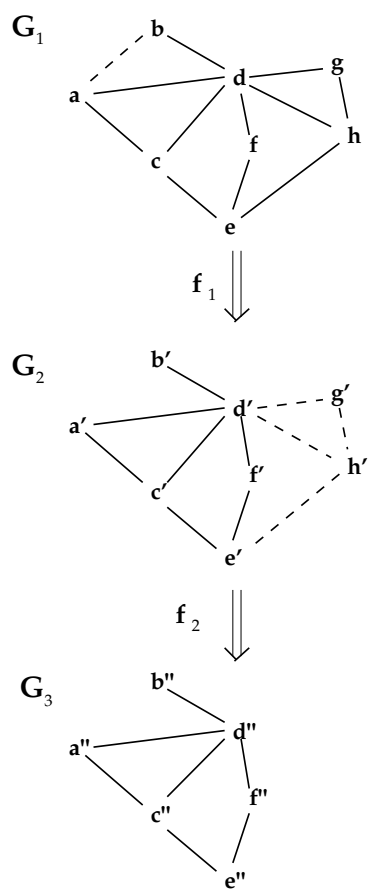

Figure 6: Contraction of a network by two successive deletions.

mation $f_{2}$ illustrates that rather large network changes can be continuous, since by Proposition 3.11 both $\delta_{g^{\prime}}$ and $\delta_{h^{\prime}}$ are continuous, and by Proposition $3.1, G_{2} . \delta_{g^{\prime}} . \delta_{h^{\prime}}$ must be continuous as well. However, removal of either connection $\left(d^{\prime}, g^{\prime}\right)$ or $\left(g^{\prime}, h^{\prime}\right)$ individually would be discontinuous. By Prop. 3.1 the composition of continuous transformations must be continuous; but as $f_{2}$ illustrates, a continuous transformation need not be decomposable into primitive continuous steps.

\section{Continuity in Practice}

It is difficult to find explicit examples of network change in the literature. A sequence of evolving networks is clearly the basis of Kossinets and Watts work in [21]. Yet, we are given only the average properties of these successive net- 
works. And, as they observe "the relative stability of average network properties, however, does not imply equivalent stability of individual network properties, for which the empirical picture is more complicated" (p.89). Therefore, in this section we turn to a network transformation that is not the result of an external social process, but rather its own internal structure.

\subsection{Network Reduction}

The networks, or undirected graphs, that normally illustrate papers such as this, are essentially trivial. They have less than 50 elements, so their structure is easily visualized and comprehended. When networks have hundreds of elements visual analysis is at best difficult [10]. One solution is to reduce its structure to some essential components and/or selected numerical characteristics. Both statistical and eigenvector analysis are often used to this end. One can use these techniques to calculate expected distance between points [7] or community structure [29]. We instead will use closed sets to simplify the structure itself.

In Figure 2 of Section 2.1, we observed that the point $c$ is not closed, that $a$ and $b$ are elements of $c . \varphi_{\eta}$. Although $\{a\}$ and $\{b\}$ are themselves closed sets, they must be contained in any closed set containing $c$. We say a point $z$ is subsumed by a set $Y$ if $z$ is an ordinary point of $Y$, that is (by Prop. 2.5) if $z . \rho \subseteq Y$. $\rho$. For the reduction process we describe below we will only consider singleton sets $Y$, such as $\{c\}$. In a sense, subsumed points such as $a$ and $b$ of Figure 2 contribute little to the closure structure, or topology, of the network. They can be eliminated with little loss of structural information.

In [36], Richards and Seay provide a small 18 point network called the "Sampson" data. They use it to contrast various eigenvector algorithms; we will use it to illustrate graph reduction by point subsumption. Figure $7(\mathrm{a})$ is one visualization of this network. The circled points of Figure 7(b) denote all the points that are subsumed by other singleton sets. For example, 7 is subsumed

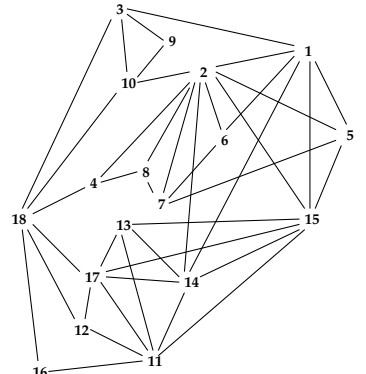

(a)

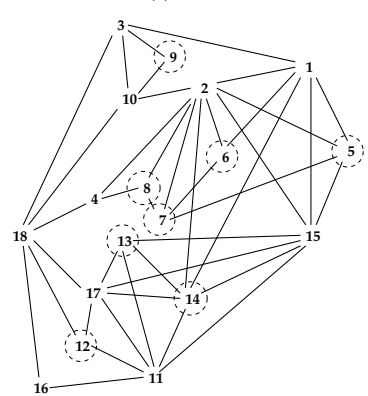

(b)

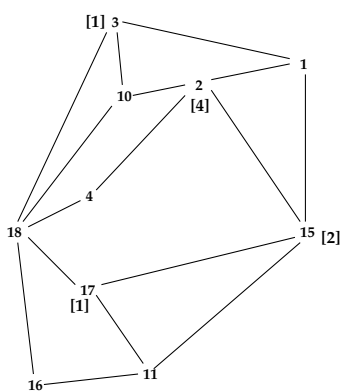

(c)

Figure 7: (a) Original "Sampson" network, (b) subsumed points, (c) reduced network.

by 2,14 is subsumed by 15 . Finally, Figure 7 (c) is the reduced graph created by deleting all subsumed points.

The reduced graph of Figure $7(\mathrm{c})$ is structurally simpler, yet its topology is faithful the the original. By recording [in brackets] the number of points subsumed by each individual node it also conveys a measure of the original density near that node. The key elements of Figure 7(c) 


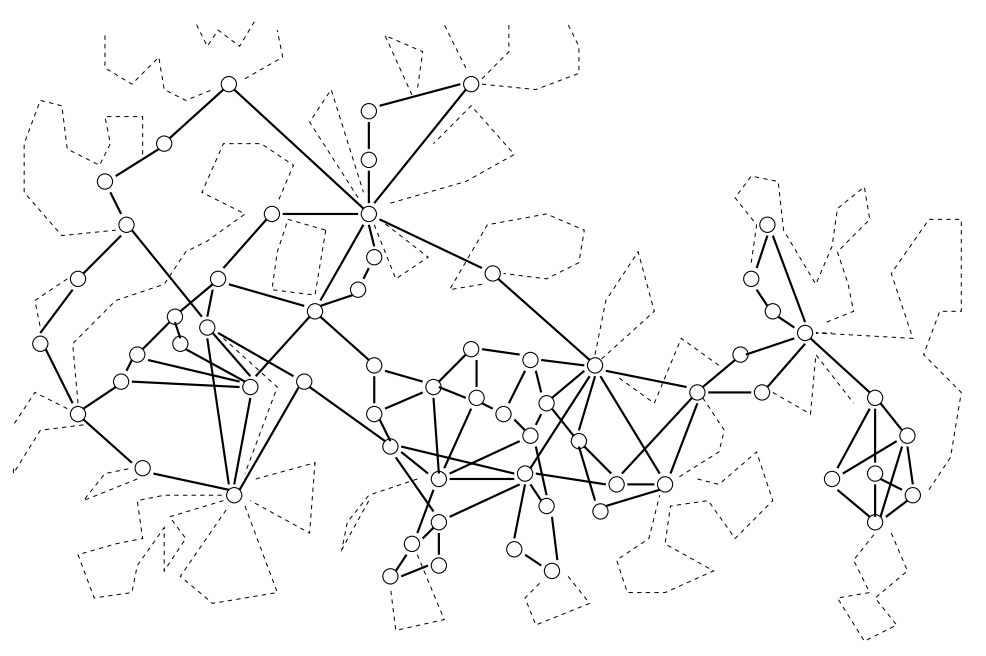

Figure 8: Chordless cycles in the collaboration network of Figure 1.

are chordless cycles of length 4 or greater. These are $\langle 3,10,2,1,3>,<18,4,2,15,17,18>$, $<2,10,18,4,2>$ and $<18,17,11,16,18>$ in the figure. ${ }^{5}$ These are chordless cycles; no point on a chordless cycle can be subsumed by another. These chordless cycles define the topology of the network in much the same manner that 1-cycles can be used to define the topological structure of manifolds [11]. By Proposition 3.11 the removal of subsumed points, such as $\delta_{7}$ in Figure 7(b) above, are each individually continuous. Thus by Proposition 3.1, their composition is continuous.

Figure 7(a) is rather simple to begin with. The continuous reduction by subsumed points is more useful in larger, more complex networks. Consider the 379 node graph of Figure 1. This was reduced by the same program that generated Figure 7(c) to the 65 node network shown in Figure 8. Dashed lines crudely approximate the extent of nodes in the original network.

In a sense, the reduction process described here is an inverse of triadic closure. It tries to remove the tight triangulated clusters of points so that the more extended connections become more evi-

\footnotetext{
${ }^{5} \mathrm{~A}$ graph, or subgraph, is said to be chordal if it contains no cycles of length greater than 3 without a chord (edge) joining two of its points $[18,23]$.
}

dent. Figure 8 still seems to have many triangles, especially in its center, but close inspection will show that every point is on some chordless cycle of length 4, or greater. The reduced representation in terms of chordless cycles is shown in Figure 8. It is a continuous image of the original 379 node network.

There is a treasure trove of sequential networks at www.boardsandgender.com/data.php describing individual participation on Norwegian corporate boards of directors. ${ }^{6}$ This is a monthly sequence; for this paper we used August 2011. Application of this reduction process to the 1421 node data set reveals that this network consists of 126 connected components. The largest of these, and only one of any interest, is shown as Figure 1 in Opshal [30]. Our process reduced it to the 103 nodes shown in Figure 9. Each node is labeled with the number of nodes it subsumes. Those nodes which have many subsumed nodes, such as [67] suggest the presence of "communities" [9]. The actual community structure can be refined using local methods [4, 41]. Extended chordless cycles, such as the emboldened path $<1-2-7-1-37>$ in the lower right sec-

\footnotetext{
${ }^{6}$ This resource has been made available by Seierstad and Opshal [39].
} 


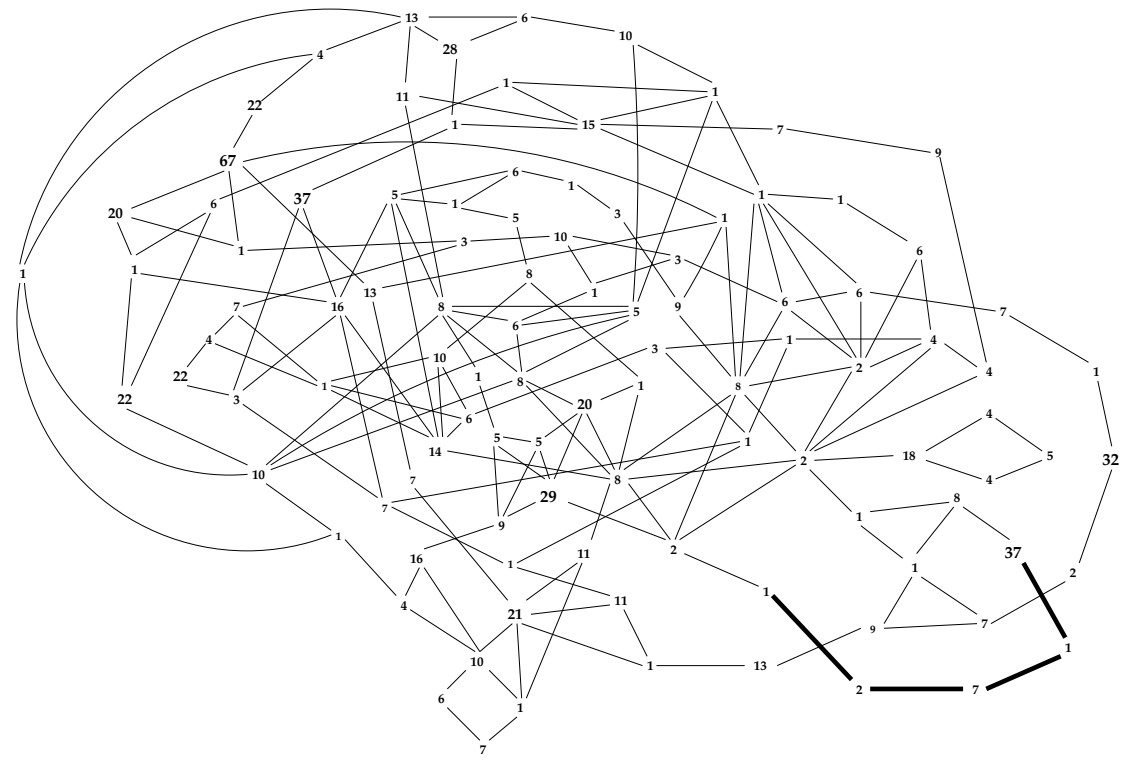

Figure 9: Chordless cycles of Norwegian directorate network.

tion invite a more careful inspection. One such is shown in Figure 10.

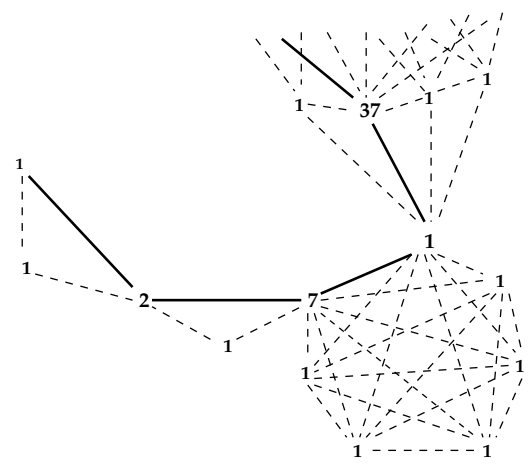

Figure 10: Expanded detail of lower right path of Figure 9

\subsection{Expanded Neighborhoods}

Throughout this paper we have assumed that the neighborhood of a set, or point, consists of those points directly connected to it by an edge in $\mathcal{A}$. And we defined the closure operator, $\varphi_{\eta}$, accordingly. But other network neighborhoods, and other network closures are possible.

Suppose we define the neighborhood of a set $Y$ to be all points connected by paths of at most 2 edges; that is $Y . \eta=\{x \mid \exists y \in Y, d(x, y) \leq 2\}$ where $d(x, y)$ denotes the "distance" between $x$ and $y$, or number of edges in a shortest connecting path $[1,15]$. Alternatively, we could use $\mathcal{A}^{2}=\mathcal{A} \times \mathcal{A}$ to represent "adjacent" points.

Regardless of the neighborhood concept, neighborhood closure is defined as in (1).

Network reduction of Figure 1 using these expanded neighborhoods yields Figure 11. Here the 65 nodes of Figure 8 have been reduced to 33 . The 32 points subsumed by these larger neighborhoods, together with their incident edges have been indicated by dashed lines. Readily, it is an even simpler rendition of Figure 1 than Figure 8.

Care must be taken when interpreting Figure 11. For the sake of clarity, the illustrated connections/edges are those of Figure 8; however the actual neighborhoods of these remaining points consist of all points one, or two, steps away. Also 


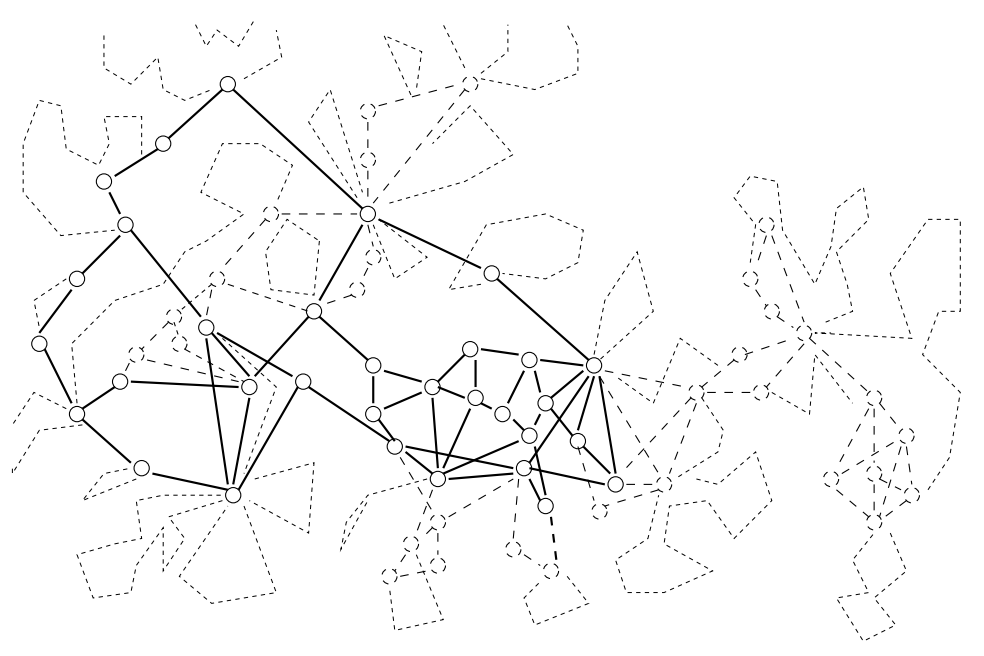

Figure 11: Newman's network reduced using expanded neighborhoods.

characterization of the chordless cycles becomes more complex. For instance, 4-cycles can no longer be chordless.

For very large networks of thousands of nodes, it is likely that expanded neighborhoods of depth $k>2$ will be needed to significantly simplify and display their structure. This remains to be explored.

\subsection{Asymmetric Neighborhoods}

We defined the "neighborhood" of a point as elements connected to it by an edge; but that is only a formal representation. The real "neighborhood" of a point, or person, are those points (persons) that are somehow "close" or "connected" to it. The edges of the network are only a formal representation of that connection. However, connections in a social network, such as "friendship", need not be bidirectional, or symmetric. We may have a "directed" edge. Neighborhoods can still be defined in terms of points that can be reached in one, or more, steps; and a neighborhood closure can be still be defined by (1). All of our development has been couched in terms of sets of neighbors without reference to specific "edges", and we know of no instance where sym- metry has been assumed. So all these results should be true for arbitrary neighborhoods, even though we have provided no examples.

The case when $\mathcal{A}$ is antisymmetric, so $\mathcal{A}$ is a partial order, and the closure is a path closure has been well studied [33]. It is the intermediate situations that are unknown, and interesting.

\subsection{Fuzzy Closure}

With neighborhood closure, as defined in Section 2.1 , a point $z$ in the neighborhood of a set $Y$ is in $Y$-closure if its neighborhood, $z . \eta$ is completely contained in $Y$. $\rho$. Thus for $z$ to be subsumed by a single point $y$, as in Section 4.1, all the neighbors/connections of $z$ must already be neighbors of $y$. This is asking for a great deal, and it is rather surprising that the form of network reduction described above works as well is it does on real networks.

When $y$ and $z$ are individuals we would be more likely to say $z$ is tightly bound to $y$ if "almost all" of $z$ 's attachments/connections/neighbors are neighbors of $y$. Can such a fuzzy concept of closure be made rigorous? 
Let us define a fuzzy neighborhood closure, $\varphi_{f}$ by $Y . \varphi_{f}=Y \cup\{w \in Y . \eta:|w . \rho-Y . \rho| \leq 1\}$, that is $w$ can have one independent attached neighbor and still be considered to be in the closure $Y . \varphi_{f}$. We use the intersection property of closure systems to show:

Proposition $4.1 \varphi_{f}$ is a closure operator.

Proof: Let $X$ and $Z$ be closed w.r.t. $\varphi_{f}$. We claim that $Y=X \cap Z$ is also closed w.r.t. $\varphi_{f}$, that is $Y . \varphi_{f}=Y$. Suppose not, then $\exists w \in(X \cap Z) . \varphi_{f}$, $w \notin X \cap z$. Let $y \in(X \cap Z) \cdot \varphi_{f}$. If $y \notin X$, there exist at least two neighbors $u, v \in y \cdot \eta, u, v \notin X$, so $u, v \notin X \cap Z$ contradicting the assumption that $y \in(X \cap Z) . \varphi_{f}$. So $y \in X$.

Assuming $y \notin Z$ leads to precisely the same contradiction, so $y \in X \cap Z$.

Readily, $Y \subseteq Y . \varphi_{\eta} \subseteq Y . \varphi_{f}$ so this fuzzy closure yields a coarser network structure. For example, the only non-trivial fuzzy closed sets of the graph of Figure 2 are $a b d$, efgh, and $h$

Because $\varphi_{f}$ is a closure operator, many of the preceding propositions are still valid; some are not. For example, the chordless cycle property (2) does not hold; $Y . \varphi_{f} \nsubseteq \subseteq$. $\rho$. If $\mathcal{S}=(\mathbf{Z}, \mathcal{A})$ with $\mathbf{Z}$ being the integers $\{1, \ldots, n\}$ and $(i, i+1) \in \mathcal{A}$, then the only closed sets are $\varnothing$ and $\mathbf{Z}$. No nonempty subset of $\mathbf{Z}$ can be closed. Because of the behavior of fuzzy closure in this last example, reduction of the network of Figure 1 using it yields only a single point! Nevertheless, the fact that one can define a fuzzy closure indicates the possibility of use in very large networks or in other kinds of social network analysis.

\section{Summary}

The results of this paper provide a rigorous mathematical foundation for studying the continuous transformation of large social networks. The characterization is based on local changes to the graph, or network, not some average global property. But, "continuity" has always been a local concept couched in terms of very small changes in the pre-image space. ${ }^{7}$ However, Proposition 3.1, the example of $f_{3}$ in Figure 4, and our application of Proposition 3.11 to network reduction demonstrate that global change, which is the composition of smaller continuous steps, may also be characterized as "continuous".

Unlike the traditional approach to continuity, the concept of the "closed set" structure of a network leads to its chordless cycles. Perhaps the

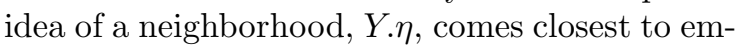
bodying the concept of "nearby points", and thus an "open" set. ${ }^{8}$ However, neighborhoods have few of the key properties of open sets, and trying to fit them into this role seems futile.

While, the introduction of closed sets to the study of transformational change has resolved a number of key issues, there are many more yet to explore. For example, suppose there exist two continuous transformations $G \stackrel{f}{\longrightarrow} G^{\prime}$ and $G^{\prime} \stackrel{g}{\longrightarrow} G$. In what way must $G$ and $G^{\prime}$ be similar, if at all? How does the chordless cycle structure change under continuous, and discontinuous, transformations? In Section 4.4, we show that a form of fuzzy closure can be defined, but we have not explored it rigorously. What properties might fuzzy continuity have?

Finally, we suspect that asking "is $f$ is continuous?" is the wrong question. It is likely that asking "why is $f$ continuous everywhere in the region $Y$, but discontinuous near its boundary?" and "what social forces cause this to happen?" will be more fruitful. In any case, it seems clear that abstract networks are a useful model of social connections and that a mathematically rigorous analysis of large social networks based on closed sets can be quite rewarding.

\section{References}

[1] Geir Agnarsson and Raymond Greenlaw. Graph Theory: Modeling, Applications and Algorithms. Prentice Hall, Upper Saddle River, NJ, 2007.

\footnotetext{
${ }^{7} E . g$. the typical $\epsilon-\delta$ definition of real analysis [37].

${ }^{8}$ Many graph theory texts say that $Y . \eta$ is an "open" neighborhood, c.f. [1, 15].
} 
[2] Kazutoshi Ando. Extreme point axioms for closure spaces. Discrete Mathematics, 306:31813188, 2006.

[3] Romain Bourqui, Frederic Gilbert, Paolo Simonetto, Faraz Zaidi, Umang Sharan, and Fabien Jourdan. Detecting structural changes and command hierarchies in dynamic social networks. In 2009 Advances in Social Network Analysis and Mining, pages 83-88, Athens, Greece, 2009.

[4] L. Karl Brantling. Context-sensitive detection of local community structure. Soc. Network Analysis and Mining, 2(3):279-289, Sept. 2012.

[5] Nicholas A. Christakis and James H. Fowler. Connected, The surprising Power of Our Social Networks and How They Shape Our Lives. Little Brown \& Co., New York, 2009.

[6] Galina Daraganova, Pip Pattison, Johan Koskinen, Bill Mitchell, Anthea Bill, Martin Watts, and Scott Baum. Networks and geography: Modelling community network structures as the outcome of both spatial and network processes. Social Networks, 34:6-17, Jan. 2012.

[7] Anthony Dekker. Conceptual Distance in Social Network Analysis. J. of Social Structure, 6(3):131,2006

[8] Paul H. Edelman and Robert E. Jamison. The Theory of Convex Geometries. Geometriae Dedicata, 19(3):247-270, Dec. 1985.

[9] Santo Fortunato. Community detection in graphs. arXiv:0906.0612v2 [physics.soc-ph], pages 1-103, Jan 2010.

[10] Linton C. Freeman. Visualizing Social Networks. J. of Social Structure, 1(1):1-19, 2000.

[11] P. J. Giblin. Graphs, Surfaces and Homology. Chapman and Hall, London, 1977.

[12] Frederic Gilbert, Paolo Simonetto, Faraz Zaidi, Fabien Jourdan, and Romain Bourqui. Communities and hierarchical structures in dynamic social networks: analysis and visualization. Soc. Networks Analysis and Mining, 1(2):83-95, April 2011.

[13] Mark S. Granovetter. The Strength of Weak Ties. Amer. J. of Sociology, 78(6):1360-1380, 1973.
[14] Nobuyuki Hanaki, Alexander Peterhansl, Peter S. Dodds, and Duncan J. Watts. Cooperation in Evolving Social Networks. Management Science, 53(7):1036-1050, July 2007.

[15] Frank Harary. Graph Theory. Addison-Wesley, 1969.

[16] Teresa W. Haynes, Stephen T. Hedetniemi, and Peter J. Slater. Fundamentals of Domination in Graphs. Marcel Dekker, New York, 1998.

[17] John R. Hipp, Robert W. Farris, and Adam Boessen. Measuring 'neighborhood': Constructing network neighborhoods. Social Networks, 34:128-140, Jan. 2012.

[18] Michael S. Jacobson and Ken Peters. Chordal graphs and upper irredundance, upper domination and independence. Discrete Mathematics, 86(1-3):59-69, Dec. 1990.

[19] Dragan Jankovic and T.R. Hamlett. New Topologies from Old via Ideals. Amer. Math. Monthly, 97(4):295-310, Apr. 1990.

[20] Gleb A. Koshevoy. Choice functions and abstract convex geometries. Mathematical Social Sciences, 38(1):35-44, 1999.

[21] Gueorgi Kossinets and Duncan J. Watts. Empirical Analysis of an Evolving Social Network. Science, 311(5757):88-90, Jan. 2006.

[22] Jure Leskovec, Kevin J. Lang, Anirban Dasgupta, and Michael W. Mahoney. Statistical Properties of Community structure in Large Social and Information Networks. In $W W W 2008$, Proc. of 17th International Conf. on the World Wide Web, pages 695-704, 2008.

[23] Terry A. McKee. How Chordal Graphs Work. Bulletin of the ICA, 9:27-39, 1993.

[24] Terry A. McKee and Fred R. McMorris. Topics in Intersection Graph Theory. SIAM Monographs on Discrete Mathematics and Applications. Society for Industrial and Applied Math., Philadelphia, PA, 1999.

[25] Gerald Mollenhorst, Beate Völker, and Henk Flap. Shared contexts and triadic closure in core discussion networks. Social Networks, 34:292302, Jan. 2012.

[26] Bernard Monjardet. Closure operators and choice operators: a survey. In Fifth Intern. 
Conf. on Concept Lattices and their Applications, Montpellier, France, Oct. 2007. Lecture notes.

[27] Bernard Monjardet and V. Raderinirina. The duality between the antiexchange closure operators and the path independent choice operators on a finite set. Math. Social Sciences, 41(2):131150, 2001.

[28] Mark E. J. Newman. The structure and function of complex networks. SIAM Review, 45:167-256, 2003.

[29] Mark. E. J. Newman. Finding community structure in networks using the eigenvectors of matrices. Phys.Rev.E, 74(036104):1-22, July 2006.

[30] Tore Opsahl. Triadic closure in two-mode networks: Redefining the global and local clustering coefficients. arXiv:1006.0887v3, 27:1-20, May 2011.

[31] Oystein Ore. Mappings of Closure Relations. Annals of Math., 47(1):56-72, Jan. 1946.

[32] John Pfaltz and Josef Šlapal. Transformations of discrete closure systems. Acta Math. Hungar., (to appear) 2012.

[33] John L. Pfaltz. Closure Lattices. Discrete Mathematics, 154:217-236, 1996.

[34] John L. Pfaltz. Logical Implication and Causal Dependency. In Henrik Schärfe, Pascal Hitzler, and Peter Øhrstrøm, editors, Conceptual Structures: Inspiration and Application, volume Springer Verlag LNAI 4068 (supplemental volume), pages 145-157, Aalborg University, July 2006.

[35] John L. Pfaltz. Establishing Logical Rules from Empirical Data. Intern. Journal on Artificial Intelligence Tools, 17(5):985-1001, Oct. 2008.

[36] William Richards and Andrew Seary. Eigen Analysis of Networks. J. of Social Structure, 1(2):1-16, 2000.

[37] H. L. Royden. Real Analysis. Mcmillian, New York, 1988.

[38] A. Saito, editor. Graphs and Combinatorics. Springer, 2010. ISSN 0911-0119.

[39] C. Seierstad and Tore Opsahl. For the few not the many? The effects of affirmative action on presence, prominence, and social capital of female directors in Norway. Scandinavian J. of Management, 27:44-54.
[40] Padhraic Smyth. Statistical Modeling of Graph and Network Data. In Proc. IJCAI Workshop on Learning Statistical Models from Relational Data, Acapulco, Mexico, Aug. 2003.

[41] Mahadevan Vasudevan and Narsingh Deo. Efficient community identification in complex networks. Soc. Network Analysis and Mining, 2(4):345-359, Dec. 2012.

[42] Josef Šlapal. A Galois Correspondence for Digital Topology . In K. Denecke, M. Erné, and S. L. Wismath, editors, Galois Connections and Applications, pages 413-424. Kluwer Academic, Dordrecht, 2004. 